\title{
Las reacciones adversas a medicamentos: una aproximación desde el punto de vista económico
}

\begin{abstract}
Omar Segura ${ }^{1}$, Carlos E. Maldonado ${ }^{2}$
1 Grupo de Farmacovigilancia, Instituto Nacional de Vigilancia de Medicamentos y Alimentos, INVIMA, Bogotá, D.C., Colombia.

${ }^{2}$ Facultad de Medicina, Universidad Nacional de Colombia, Bogotá, D.C., Colombia.

La farmacovigilancia, en cuanto disciplina y actividad de las ciencias de salud dedicada a la recolección de información, análisis y ayuda a la toma de decisiones sobre reacciones adversas a medicamentos (RAM), ha tenido expansión conceptual y práctica desde la década de 1960. No obstante, muy pocos estudios han analizado en profundidad cuánto puede costarle este problema a un sistema de salud. El presente artículo pretende ofrecer un análisis de la literatura disponible, orientado hacia el costo generado por la atención de eventos adversos frente a medicamentos en la práctica médica - basado en datos de la literatura internacional - y ofrece un ejercicio hipotético de extrapolación sobre futuros costos de las RAM en Colombia. La finalidad última del presente artículo es sensibilizar y llamar a la reflexión a los diferentes actores del sector salud sobre el impacto económico y la necesidad de apoyar la implementación y desarrollo de un sistema nacional de farmacovigilancia.
\end{abstract}

Palabras clave: farmacovigilancia, reacción adversa a medicamento, farmacoeconomía.

\section{Evaluation of medication side-effects from an economic perspective}

Pharmacovigilance is a health sciences discipline devoted to the data collection, data analysis and decision-making related to adverse drug reactions (ADR). It has played an expanded theoretical and practical role since the 1960's. However, few studies have made a careful analysis of the decision-making costs in evaluating ADRs. Herein, the relevant literature is reviewed concerning the costs generated due to the attention of the drug adverse events in medical practice. Examples are taken from international literature which offer by extrapolation of the potential future costs of ADR in Colombia. The objective is to sensitize and generate insights about the need for implementation and development of a national pharmacovigilance system.

Key words: pharmacovigilance, adverse drug reaction, pharmacoeconomics.

Los medicamentos han sido factor clave en la prolongación de la esperanza de vida de la población, el control de los costos médicos, la erradicación o el control de ciertas enfermedades y el bienestar general de la población (1-2). No obstante, para los profesionales de salud, la necesidad de hacer farmacovigilancia es aún un tema nuevo. Los conceptos de farmacovigilancia

Correspondencia:

Omar Segura, Calle 74 No.72-11, Bogotá, D.C., Colombia. Teléfono: (571) 543 1970; fax: (571) 294 8700, ext. 3917. osmdmsc@tutopia.com

Recibido: 21/04/03; aceptado: 3/10/03 (disciplina de las ciencias de la salud dedicada a la recolección de información, el análisis y la ayuda a la toma de decisiones acerca de eventos adversos a medicamentos) y los de reacción o evento adverso a los medicamentos tienen su expresión moderna desde fines de la década de $1960(3,4)$ y en la existencia de agencias reguladoras que controlen estándares de calidad, seguridad y eficacia durante la vida útil de los medicamentos.

Desde el Proyecto Colaborativo de Vigilancia de Medicamentos de Boston, a comienzos de la década de 1970, las reacciones adversas a 
medicamentos, es decir, todos aquellos efectos indeseados, inesperados, con asociación temporal, que ocurren con medicamentos usados a las dosis recomendadas por el fabricante $(1,5)$ han llegado a ser reconocidas como una causa importante de hospitalizaciones, con una proporción que varía entre 0,9 y $28 \%$ (6-8), de entre $1,5 \%$ y $43,5 \%$ en casos intrahospitalarios (6) y una incidencia en población general de 3,2\%; las reacciones adversas a medicamentos son igualmente responsables de un número importante de muertes - tantas como 140.000/año en los Estados Unidos $(9,10), 2 \%$ a $12 \%$ de todas las muertes hospitalarias - y de un significativo aumento de los costos de salud, principalmente en términos de estancia hospitalaria, exámenes paraclínicos, medicamentos y costos indirectos (11). Factores tales como frecuencia, severidad, causalidad y posibilidad de prevención empiezan a ser objeto de preocupación entre investigadores (12).

La farmacovigilancia se apoya, entre otras metodologías, en el análisis de reportes espontáneos de reacciones adversas a medicamentos (RAM). Aunque es un sistema eficiente de detección de nuevos problemas en medicamentos comercializados, necesita el complemento de otras metodologías cuando se trata de realizar estudios de impacto económico de esas reacciones. Pero aún hay más: resulta interesante constatar la relativa consistencia y el sitio de ocurrencia en las anteriores cifras: estudios diversos alrededor del mundo (13-21), han sido enfocados sobre aspectos del medicamento que van desde descripción epidemiológica, pasan por evaluación de grupos farmacológicos, número de medicamentos recibidos, factores de riesgo de ocurrencia, para llegar a protocolos de evaluación e, incluso, pruebas alfa y beta de bases computarizadas hospitalarias. Sin embargo, muy pocos estudios $(9,17)$ han analizado en profundidad cuánto puede costarle a un sistema de salud el problema de las RAM: problemas de heterogeneidad geográfica, cultural e, incluso, coyuntural han llevado en este tema a resultados dispares, con amplia dispersión y considerable margen de error.

El presente artículo pretende ofrecer una revisión de la literatura disponible dirigida hacia el análisis del costo generado por la atención de eventos adversos frente a medicamentos en la práctica médica - basado en datos de la literatura internacional - y hacer un ejercicio hipotético de extrapolación sobre futuros costos de las RAM en Colombia, cuya finalidad es sensibilizar a los diferentes actores del sector salud sobre la necesidad de apoyar la implementación y el desarrollo de un sistema nacional de farmacovigilancia.

\section{Metodología}

Se realizó, entre noviembre 01 de 2002 y marzo 31 de 2003, una búsqueda de literatura con operadores booleanos usando los términos "pharmacoepidemiology", "pharmacovigilance", "adverse drug reaction", "pharmacoeconomics", "cost", "economics", utilizando la base de datos Medline, y con los términos "farmacoepidemiología" o "farmacovigilancia", mediante la base de datos Lilacs, para localizar aquellos artículos publicados sobre el tema entre 1988 y 2003 , dado que en este lapso de tiempo el tema ha tenido mayor expansión o difusión $(1,9,11)$. Se contó, además, con el recurso de la iniciativa HINARI (Health Internetwork Research Initiative), de la OMS. Se agruparon los artículos en dos grupos: aquéllos que fuesen revisiones de tema, y aquéllos que fuesen series de casos, estudios analíticos, experimentales, o metanálisis. De este segundo grupo fueron extraídos datos correspondientes a variables cuantitativas relacionadas con mortalidad, morbilidad, sexo y edad de pacientes, costo por hospitalización y costo extra de atención por RAM, así como el tipo de algoritmo utilizado para clasificar las reacciones. Para el cálculo del costo corregido en dólares, se asumió una depreciación del dólar estadounidense del $7 \%$, anual y constante. Para dichos datos se realizó un análisis estadístico utilizando medidas descriptivas de tendencia central y de dispersión, usando la hoja de cálculo Excel 2000.

Luego de obtener esta información, se realizó un ejercicio de extrapolación para el caso colombiano desde el año 2002 hasta el 2012, para hacer un cálculo aproximado en un escenario donde no exista el estudio de las reacciones adversas a 
medicamentos. Para tal fin, se asumieron los siguientes supuestos: una inflación uniforme del $10 \%$ anual, un número de 5.000 instituciones prestadoras de servicios de salud (IPS) de nivel I, II y III - superior al presentado en un informe reciente (22) -, una incidencia de una a tres reacciones adversas a medicamento reportadas cada año por cada IPS en el país, un costo de atención hospitalaria de COL $\$ 11^{\prime} 000.000 /$ día, valor asumido para el año 2003 a partir de datos de un análisis de costo nacional (23), y una RAM moderada que genere una hospitalización extra de 5 días.

\section{Resultados}

Entre 2.350 artículos encontrados, se seleccionaron 36 artículos compatibles con la búsqueda específica, de los cuales 24 pertenecían a la primera categoría y 12 a la segunda. Con esos 12 artículos se analizaron las variables reseñadas en el cuadro 1. Los datos encontrados sugieren un tiempo de estudio promedio de un año, principalmente estudios descriptivos y, en menor medida, estudios analíticos; nivel institucional de alta especialización reportado en 10 de 12 estudios, un tiempo extra de estancia

Cuadro 1. Parámetros de estancia, costo, morbimortalidad por artículo seleccionado.

\begin{tabular}{|c|c|c|c|c|c|c|c|c|c|}
\hline Estudio & Tipo & Año & $\begin{array}{l}\text { Tiempo } \\
\text { (meses) }\end{array}$ & Nivel & \multicolumn{2}{|c|}{ Admisiones } & $\begin{array}{c}\text { Tiempo } \\
\text { de estancia }\end{array}$ & $\begin{array}{c}\text { Tiempo } \\
\text { extra } \\
\text { de estancia }\end{array}$ & $\begin{array}{c}\text { Costo extra } \\
\text { corregido } \\
\text { US\$ }\end{array}$ \\
\hline Classen & caso-control & 1997 & 36 & 3 & \multicolumn{2}{|c|}{91.574} & 7,69 & 1,74 & $3.705,392$ \\
\hline Gholami & experimento & 1999 & 9 & 3 & \multicolumn{2}{|c|}{370} & ND & ND & ND \\
\hline Lacoste-Rousillon & serie casos & 2001 & 2 & 1 & \multicolumn{2}{|c|}{13} & ND & ND & ND \\
\hline Bates & caso-control & 1999 & 6 & 3 & \multicolumn{2}{|c|}{3.848} & 12 & 6 & ND \\
\hline Dong & caso-control & 2000 & 6 & 3 & \multicolumn{2}{|c|}{9.311} & 10,6 & 6,8 & $10.801,51$ \\
\hline Couffignal & serie casos & 2000 & 12 & 3 & \multicolumn{2}{|c|}{4.619} & 5,1 & 3,1 & $1.859,68$ \\
\hline Nelson & serie casos & 1996 & 1 & 3 & \multicolumn{2}{|c|}{452} & 4 & 2 & ND \\
\hline Chyka & estad nacional & 2000 & 12 & nacional & \multicolumn{2}{|c|}{ 2'312.203 } & ND & ND & ND \\
\hline Ibañez & serie casos & 1991 & 33 & 3 & \multicolumn{2}{|c|}{48.678} & ND & ND & ND \\
\hline Moore & serie casos & 1998 & 6 & 3 & \multicolumn{2}{|c|}{329} & 19 & 8,5 & ND \\
\hline Bordet & serie casos & 2001 & 18 & 3 & \multicolumn{2}{|c|}{16.916} & 11 & 4 & 8.715 \\
\hline Bates (JAMA) & cohorte & 1997 & 6 & 3 & \multicolumn{2}{|c|}{4.108} & 20,4 & 2,2 & $5.222,84$ \\
\hline Promedio & & & 12,25 & & 207.701 &, 75 & 11,22 & 4,29 & $5.050,74$ \\
\hline Mediana & & & 7,5 & & 4.36 & 3,5 & 10,8 & 3,55 & $4.464,12$ \\
\hline DE & & & 11,39 & & 663.310 & 17 & 5,95 & 2,52 & $4.099,53$ \\
\hline Estudio & $\begin{array}{c}\text { Casos de } \\
\text { RAM }\end{array}$ & $\begin{array}{l}\text { Proporción } \\
\text { mortalidad }\end{array}$ & Edad & $\begin{array}{r}\text { Raz } \\
\text { hombr }\end{array}$ & $\begin{array}{l}\text { zón } \\
\text { re/mujer }\end{array}$ & $\begin{array}{c}\text { Casos } \\
\text { leves }\end{array}$ & $\begin{array}{c}\text { Casos } \\
\text { moderados }\end{array}$ & $\begin{array}{l}\text { Casos } \\
\text { graves }\end{array}$ & Algoritmo \\
\hline Classen & 0,024 & 0,035 & 54 & & JD & 0,0202 & 0,921 & 0,058 & Naranjo \\
\hline Gholami & ND & ND & 56 & & JD & 0,098 & 0,869 & 0,029 & Naranjo \\
\hline Lacoste-Rousillon & 0,846 & 0,00157 & 56 & 0,6 & 615 & & 0 & 1,000 & Turner \\
\hline Bates & ND & ND & 51,8 & 0,4 & 432 & & 0,854 & 0,146 & TISS \\
\hline Dong & 0,021 & 0,02 & 56 & 0,5 & 504 & 0,3 & 0,53 & 0,170 & Naranjo \\
\hline Couffignal & 0,062 & ND & 54,9 & 0,3 & 359 & ND & ND & ND & ND \\
\hline Nelson & 0,162 & ND & 43 & &, 6 & ND & ND & ND & Naranjo \\
\hline Chyka & 0,002 & 0,00009 & 60 & 0,5 & 539 & ND & ND & ND & ND \\
\hline Ibáñez & 0,011 & 0,028 & 69,5 & 0,7 & 714 & 0 & 0,825 & 0,175 & Lasagna \\
\hline Moore & 0,094 & 0 & 57,2 & 0,5 & 551 & 0 & 0 & 1,000 & ND \\
\hline Bordet & 0,022 & 0,048 & 59 & & 55 & 0 & 0,64 & 0,360 & WHO \\
\hline Bates & 0,046 & 0,048 & 55,6 & 0,5 & 516 & 0,57 & 0,3 & 0,130 & TISS \\
\hline Promedio & 0,13 & 0,02 & 56 & &, 54 & 0,11 & 0,55 & 0,34 & \\
\hline Mediana & 0,035 & 0,02 & 56 & & 54 & 0 & 0,64 & 0,17 & \\
\hline DE & 0,26 & 0,02 & 6 & & 10 & 0,20 & 0,37 & 0,38 & \\
\hline
\end{tabular}


hospitalaria cercano a una semana laboral (cinco días) y un costo extra por cada RAM cercano a Col $\$ 15$ millones en 2003. Adicionalmente, fueron evidentes un promedio de 13 casos de RAM por cada 100 pacientes hospitalizados, de los cuales murieron entre uno y dos pacientes, ocurrencia de RAM ligeramente mayor en hombres entre 40 y 70 años de edad. Es importante señalar que, en promedio, 8 de cada 10 reacciones adversas a medicamentos fueron moderadas o severas, pero no leves, y el algoritmo de clasificación de reacciones adversas a medicamento más utilizado fue el de Naranjo (5) en 4 de 12 casos; este algoritomo es el más utilizado para establecer relaciones de causalidad entre un evento adverso y los medicamentos.

Los datos de la extrapolación se presentan en la figura 1. Bajo los supuestos arriba señalados, el costo de las reacciones adversas a medicamentos puede actualmente oscilar entre Col\$25 millardos y Col\$ 75 millardos; hacia 2010, tanto el costo real de un día de atención hospitalaria como el costo derivado de la presentación de reacciones adversas a medicamentos se habrán duplicado (entre \$ 53 millardos y $\$ 160$ millardos).

\section{Discusión}

La razón de ser de la farmacovigilancia es identificar eventos adversos, analizarlos y generar estrategias de prevención; tal actividad se puede complementar por análisis de costos, dado que el concepto económico de este problema es un

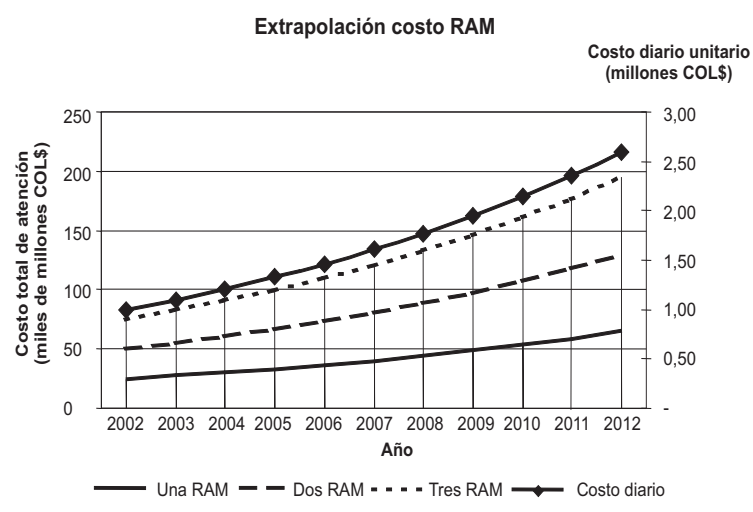

Figura 1. Extrapolación para los próximos 10 años, del costo diario de atención de una RAM y del costo total de atención de RAM en Colombia. enfoque incipiente en nuestro medio. El impacto de prevenir eventos adversos, según la presente revisión de la literatura, significa una reducción de costos de atención médica hasta en un $60 \%$ de los costos actuales (24-27). Ahora, de 11 estudios evaluados cuantitativamente, 9 sugieren atención en el nivel 3, es decir, especializado. Esto sugiere varias cosas: 1) que el problema de reacciones adversas a medicamentos consume recursos y profesionales de nivel especializado; 2) que hay una necesidad sentida de cobertura del problema al nivel de atención primaria; 3) que se requiere educación continuada y conocimiento adicional para detectar y manejar estas reacciones. Dada una morbilidad hospitalaria del $14 \%$ y una mortalidad hospitalaria del $2 \%$, detectada como promedio en los estudios de farmacovigilancia, este dato puede equivaler a un problema de salud pública $(28,29)$.

En un estudio prospectivo de dos meses (11) se estudiaron los eventos adversos ocurridos durante cinco días de un médico general dado, y se encontró una densidad de incidencia de RAM de 10,2 por 1.000 días de práctica. Esto, teniendo presente reacciones adversas a medicamento confirmadas y validadas, significa que por cada RAM confirmada hay muchos más eventos adversos a medicamentos, hecho confirmado también por otros investigadores $(30,31)$. Las RAM pueden causar incrementos significativos de la morbimortalidad de los pacientes, en especial aquellos hospitalizados. Sin embargo, la mayoría de las muertes derivadas de reacciones adversas a medicamentos tienen factores de confusión o modificadores del efecto, tales como vejez, comorbilidad, polifarmacia y, sólo en parte, inapropiada prescripción o uso de medicamentos $(8,19,21)$. Suele existir más subregistro de RAM en casos leves a moderados, lo que no significa que sea inútil como sistema para la detección de problemas severos $(18,21,32)$.

En el presente análisis, el promedio del costo extra por RAM es relativamente más bajo pese a un mayor tiempo de estancia. Esto tiene explicación si consideramos que la mayor parte de los estudios disponibles para análisis cuantitativo no provenían de los Estados Unidos, donde la atención en salud es relativamente 
costosa. En un estudio (12), todos los eventos adversos fueron filtrados por un algoritmo para establecer causalidad, y pasó por un proceso adicional para establecer su carácter de severidad. Teniendo en cuenta los costos directos, concluyó que los costos adicionales en pacientes que sufrieron reacciones adversas cutáneas severas asociadas con el uso de antiepilépticos fue de US\$ 8.032/paciente/año. Del mismo modo, otro estudio (1) mostró que en un hospital universitario con 520 camas, los costos para pacientes con RAM durante su hospitalización alcanzaron US $\$ 3,2$ millones, durante el período de estudio de 4 años. Hay que tener presente que los costos directos son costos de diagnóstico y tratamiento, salario a profesionales de salud y costos de hospitalización; así mismo, hay otras medidas de impacto económico en salud, tales como AVISA (Años de Vida Saludable Perdidos), QALY y DALY (Años de Vida Asociados con Calidad de Vida o con Inhabilidad), que valdría tener en cuenta en subsiguientes investigaciones sobre el tema en Colombia.

Dado que las RAM resultan en efectos transitorios o, más comúnmente, morbilidad persistente o mortalidad, aparte del impacto personal y familiar, también ha de considerarse el que sufre la sociedad. Desde 1997, estudios sistemáticos se han aproximado al estudio del impacto económico de los fenómenos adversos a medicamentos, aunque sin contar con una metodología estándar plenamente difundida. Por lo menos, un trabajo estadounidense (17) ha sugerido que hasta el $50 \%$ (rango 30\% a 70\%) de las reacciones adversas a medicamentos probablemente se hubieran evitado, ya que las mismas están relacionadas con las propiedades farmacológicas y el mecanismo de acción del medicamento (reacciones tipo A); en Europa, Detournay (20) ha sugerido que el $60 \%$ de las RAM sí son prevenibles y que pueden desarrollarse estrategias para lograrlo; igualmente, un modelo para evaluar la factibilidad de prevención de reacciones adversas (30) encontró que, de 44 tipos de reacción adversa, $25 \%$ eran potencialmente prevenibles. Este tipo de estudios, no obstante, deben ser validados y contextualizados frente a la delicada situación actual del sector salud colombiano.
En la implementación de estudios de farmacovigilancia, los estudios dirigidos hacia varios medicamentos y varias enfermedades pueden ser más productivos y más económicos que muchos estudios orientados hacia una patología o un medicamento en particular, pero el diseño del estudio es fundamental para la validez de los resultados $(12,33,34)$. Existen, de hecho, diferencias por servicios: la atención de un paciente con una RAM moderada en oncología (20) puede costar entre US\$1.400 y US\$4.000. Es de resaltar también que existen diferencias entre los reportantes (sean o no profesionales de salud); Ottervanger (35) reportó que el $54 \%$ de los pacientes reportaron cualquier RAM, mientras que sólo el $11 \%$ de los médicos generales reportaron RAM de los pacientes. En este estudio, la diferencia en el porcentaje de reportes se explica en que al ser recibido el reporte de reacción adversa al médico, éste no lo registra o no lo informa; razones tales como falta de conocimiento, subvaloración del reporte o factores de confusión (uso de más de un medicamento que genere el mismo problema) o modificación del efecto (problemas de interacción) pueden influir en el subregistro. Dado el bajo número de reportes de reacción adversa a medicamentos, el paciente reporta mucho más eventos adversos que lo que reporta el médico a un sistema de farmacovigilancia; esta posibilidad de incluir al paciente en el sistema de reporte funciona usualmente a través de dos vías: 1) que el paciente reporte a su profesional de salud el problema para que, a su vez, el formato de reporte sea diligenciado y enviado, o bien 2) enviar un reclamo a una oficina de quejas y reclamos estatal, para que el organismo pertinente esté enterado del caso.

Durante la vida comercial de un medicamento, siempre se están buscando respuestas sobre parámetros que normalmente son asumidos dentro del llamado "ciclo sanitario del medicamento" (cuadro 2). Al comenzar a hacer farmacovigilancia, se detectan problemas tales como prescripción inadecuada, administración, dosis y vía de administración inadecuadas, poca profundización en la historia farmacológica de los pacientes, monitoreo inadecuado de medicamentos y otras pruebas de laboratorio, carencia de evaluación de 
Cuadro 2. Temas de interés para investigación en farmacoepidemiología y farmacovigilancia.

- Estudios o experiencias en grupos especiales

- Identificación de factores de riesgo en el uso de medicamentos

- Evaluación de interacciones entre medicamentos y con alimentos

- Ampliación o restricción de indicaciones terapéuticas

- Análisis de efectos secundarios a corto y largo plazo

- Cambios en la posología de medicamentos

- Seguimiento a seguridad y toxicidad a largo plazo

- Detección de efectos benéficos inesperados

- Evaluación farmacoeconómica de costos vs. calidad de vida

interacciones medicamentosas, desconocimiento de conceptos en farmacocinética y farmacodinamia. Por consiguiente, debieran ser mandatorios la educación continuada a los profesionales en salud, así como sistemas de farmacovigilancia en las instituciones de salud, que reporten a una entidad central experta en el estudio y manejo de estos problemas.

Con este panorama, y con las limitaciones implícitas en los estudios de costos, consideramos que se justifican todos los esfuerzos orientados a implementar tanto el programa nacional de farmacovigilancia como la educación continuada al cuerpo de profesionales de salud así como investigación y desarrollo en el tema, sostenidos todos estos elementos por una regulación estatal. Actualmente, el sector salud en Colombia debe replantearse de tal forma que disciplinas tales como la farmacoepidemiología (con la farmacovigilancia como una de sus actividades), la farmacoeconomía y la economía en salud tengan muy presente el beneficio del paciente, con sanos criterios de costo, utilidad, eficiencia y efectividad, especialmente este último (36-39). Estas disciplinas son dinámicas, están en proceso de evolución y los encargados de desarrollar políticas en salud deben entender la importancia de aplicarlas y contextualizarlas al entorno. Tales disciplinas, tales conceptos, permiten la planeación a corto, mediano y largo plazo (13), a diferencia de las medidas de emergencia basadas únicamente en precios.

Los fenómenos adversos a medicamentos son costosos tanto en forma directa (sobre el bienestar del paciente) como en forma indirecta (sobre el sistema de salud y la sociedad) y las intervenciones dirigidas a reducir su frecuencia están justificadas tanto desde la calidad del cuidado de salud como desde el aspecto meramente económico $(24,25,28,33,34)$. Con carácter urgente, se requieren más estudios en esta disciplina, en especial en el medio y circunstancias latinoamericanas, para establecer nuevas líneas de trabajo orientadas al desarrollo de modelos de detección, análisis y prevención de las RAM.

Declaración de conflicto de intereses: ninguno.

\section{Referencias}

1. Classen DC, Pestonik SL, Evans RS, Lloyd JF, Burke JP. Adverse drug events in hospitalized patients. Excess length of stay, extra costs and attributable mortality. JAMA 1997;277:301-3.

2. Nelson KW, Talbert RL. Drug-related hospital admissions. Pharmacotherapy 1996;16:701-7.

3. Laporte JR, Lience E. Información mínima que deben contener las publicaciones sobre sospechas de reacciones adversas a medicamentos. Med Clin (Barc) 1991;97:56-7.

4. Karch FE, Lasagna L. Adverse drug reactions. A critical review. JAMA 1975;234:1236-41.

5. Naranjo CA, Shear NH, Lanctôt KL. Advances in the diagnosis of adverse drug reactions. J Clin Pharmacol 1992;32:897-904.

6. Gholami K, Shalviri G. Factors associated with preventability, predictability and severity of adverse drug reactions. Ann Pharmacother 1999;33:236-40.

7. Suh DC, Woodall BS, Shin SK, Hermes-De Santis ER. Clinical and economical impact of adverse drug reactions in hospitalized patients. Ann Pharmacother 2000;34:1373-9

8. Ajayi FO, Sun H, Perry J. Adverse drug reactions: a review of relevant factors. J Clin Pharmacol 2000;40: 1093-101.

9. Bates DW, Spell N, Cullen DJ, Burdick E, Laird N, Petersen LA, et al. The costs of adverse drug events in hospitalized patients. JAMA 1997;277:307-11.

10. Jeffrey White T, Arakelian A, Rho JP. Counting the costs of drug-related adverse events. Pharmaco-economics 1999;15:445-58.

11. Bates DW, Miller EB, Cullen DJ, Burdick L, Williams L, Laird N, et al. Patient risk factors for adverse drug events in hospitalized patients. Arch Int Med 1999;159: 2553-60. 
12. Lazarou J, Pomeranz BH, Corey PN. Incidence of adverse drug reactions in hospitalized patients - a metaanalysis of prospective studies. JAMA 1998;279: 1200-5.

13. Lacoste-Rousillon C, Pouyanne P, Haramburu F, Miremont G, Bégaud B. Incidence of serious adverse drug reactions in general practice: a prospective study. Clin Pharmacol Ther 2001;69:458-62.

14. Couffignal AL, Lapeyre-Mestre M, Bonhomme C, Bugat R, Montastruc JL. Effets indésirables des médicaments anticancéreux: à propos d'une étude de pharmacovigilance au sein d'une institution spécialisée en cancérologie. Thérapie 2000;55:635-41.

15. Chyka PA. How many deaths occur annually from adverse drug reactions in the United States? Am J Med 2000;109:122-30.

16. Ibañez L, Laporte JR, Carné $\mathbf{X}$. Adverse drug reactions leading to hospital admission. Drug Safety 1991; 6:450-9.

17. Moore N, Lecointre D, Noblet C, Mabille M. Frecuency and cost of serious adverse drug reactions in a department of general medicine. Br J Clin Pharmacol 1998;45:301-8.

18. Bordet R, Gautier S, Le Louet H, Dupuis B, Caron J. Analysis of the direct cost of adverse drug reactions in hospitalised patients. Eur J Clin Pharmacol 2001;56: 935-41.

19. Jick H. Adverse drug reactions: the magnitude of the problem. J Allergy Clin Immunol 1984;74:555-7.

20. Detournay B, Fagnani F, Pouyanne P, Haramburu F, Bégaud $B$, Welsch $M$, et al. Coût des hospitalisations pour effet indésirable médicamenteux. Thérapie 2000; 55:137-9.

21. Ioannides-Demos LL, Eckert GM, McLean AJ. Pharmacoeconomic consequences of measurement and modification of hospital drug use. Pharmaco-economics 1992;2:15-33.

22. www.paho.org/english/sha/prflcol.htm

23. http://colombiamedica.univalle.edu.co/Vol33No2/ cm33n2a1.htm

24. Winterstein AG, Hatton RC, González-Rothi R, Johns TE, Segal R. Identifying clinically significant preventable adverse drug events through a hospital's database of adverse drug reaction reports. Am J Health Syst Pharm 2002;59:1742-9.

25. Bongard V, Ménard-Taché S, Bagheri $H$, Kabiri $K$, Lapeyre-Mestre M, Montastruc JL. Perception of the risk of adverse drug reactions: differences between health professionals and non health professionals. $\mathrm{Br}$ J Clin Pharmacol 2002;56:433-6.
26. Lehmann DF. Observation and experiment on the cusp of collaboration: a parallel examination of clinical pharmacology and pharmacoepidemiology. J Clin Pharmacol 2000;40:939-45.

27. Spreux A, Chichmanian RM, Castot A. Impact économique des effets indésirables des médicamentsquelques réflexions. Thérapie 1992;47:385-8.

28. MacLeod SM. Pharmacoepidemiology: a health imperative. J Clin Epidemiol 1991;44:1285-6.

29. Walley T. Drugs, money and society. Br J Clin Pharmacol 1995;39:343-5.

30. Olivier $\mathbf{P}$, Boulbes $\mathbf{O}$, Tubery $\mathbf{M}$, Lauque $\mathbf{D}$, Montastruc JL, Lapeyre-Mestre M. Assessing the feasibility of using an adverse drug reaction preventability scale in clinical practice. A study in a French emergency department. Drug Safety 2002;25:1035-44.

31. Mjordal T, Boman MD, Hagg S, Backstrom M, Wiholm BE, Wahlin A, et al. Adverse drug reactions as a cause for admissions to a department of internal medicine. Pharmacoepidemiol Drug Saf 2002;11:65-72.

32. van den Bemt PM, Egberts AC, Lenderink AW, Verzijl JM, Simons KA, van der Pol WS, et al. Adverse drug events in hospitalized patients - a comparison of doctors, nurses and patients as sources of reports. Eur J Clin Pharmacol 1999;55:155-8.

33. Temple R. Meta-analysis and epidemiologic studies in drug development and postmarketing surveillance. JAMA 1999;281:841-4.

34. Reith $\mathbf{C H}$, Malone DC. Understanding the fundamental concepts for interpreting or conducting meta-analyses. Formulary 2001;36:594-609.

35. Ottervanger JP, Valkenburg HA, Grobbee DE, Stricker BH. Differences in perceived and presented adverse drug reactions in general practice. J Clin Epidemiol 1998;51:795-9.

36. Hasford J, Goettler M, Munter KH, MullerOerlinghausen B. Physicians' knowledge and attitudes regarding the spontaneous reporting system for adverse drug reactions. J Clin Epidemiol 2002;55:945-50.

37. Lapeyre-Mestre M, Gary J, MachelardRoumagnac $\mathbf{M}$, Bonhomme $\mathbf{C}$, Bugat $\mathbf{R}$, Montastruc JL. Incidence and cost of adverse drug reactions in a French cancer institute. Eur $\mathrm{J}$ Clin Pharmacol 1997;53:19-22.

38. Abadie E, Souetre E. Aspects économiques de la pharmacovigilance dans l'industrie pharmaceutique. Thérapie 1993;48:125-7.

39. Faich GA. Pharmacoepidemiology and clinical research. J Clin Epidemiol 1991;44:821-2. 